\title{
Implementation Basic Network Design with Netkit For Evaluation of Network Learning
}

\author{
https://doi.org/10.3991/ijoe.v15i08.9795 \\ Yuri Ariyanto $\left.{ }^{\bowtie}\right)$, Budi Harijanto, Yan Watequlis S. \\ State Polytechnic of Malang, East Java, Indonesia \\ yuriepolinema.ac.id
}

\begin{abstract}
A virtual laboratory with a network emulator environment using NetKit is one of series of basic network laboratories on basic computer network competencies where students are given practical trial opportunities at low costs and little effort in their implementation. Teaching computer network subjects to be easily understood by students needs an instructional media as a tool in delivering material. This media uses computer virtualization technology, i.e. creating a virtual laboratory, as a means of students in conducting experiments from the material that has been obtained. In virtual laboratories it is possible to implement network topology designs based on actual network topologies. This implementation is used as a testing tool before the network topology is implemented on the actual network. Therefore, errors can be identified first without disturbing the system that is already running. For testing, the students are given training using a basic network design consisting of the implementation of routing tests, firewalls, ftp server implementation and web server. This paper is aimed at describing ways to develop a virtual laboratory with a network emulator environment using NetKit. Moreover, several exercises on network topology implementation that are applied directly to the real world with NetKit are introduced, such as describing laboratory settings, describing the main parts of the lab, illustrating lab instructions, and reporting lab feeds.
\end{abstract}

Keywords - Virtual Laboratories, Learning Media, Computer Networks, Network Design, Netkit.

\section{$1 \quad$ Introduction}

In the era of the development of virtualization technology, network virtualization plays an important role in its use as a learning medium. In addition, network virtualization is effectively adopted in many other contexts where the function of the emulator is useful as a test before implementation, scenario evaluation, research trials. [1]

This paper describes the writers'experiences in teaching computer network material, where students often experience difficulties in understanding the given materials. The writers use Netkit as a network emulator, where Netkit can do simple, cheap and 
lightweight network design, implementation and testing targeted at actual implementation.

Moreover, in this study the writers make a network topology design that describes the basic competencies that students must possess after taking part in computer network learning. To get the results of this study, a trial is conducted to obtain an analysis of the design that has been implemented based on the existing scenario. It is expected from the results of this study that teachers and students can use virtual laboratories as learning media for computer networks. Besides, the students can get an overview of the basic competencies of computer networks that must be possessed after implementing virtual laboratories using Netkit.

\section{Literature Review}

\subsection{Network emulator}

A network emulator is software in its environment mimicking the functions and habits of the original network [1].

Network emulators are implemented as machine virtualization. The use of virtualization machines in the class of computer networks, is used to provide an experimental environment in network practicums. With this network emulator, it can be used as a solution in learning computer networks [2].

\subsection{User mode linux (UML)}

User Mode Linux (UML) is a Linux virtual machine running on Linux. Technically, UML is a port connection from Linux to Linux. Linux has connected ports to many different processors, including X86, Sun's SPARC, IBM and Motorola's PowerPC, DEC's (Compaq and HP) Alpha and various other processors [3].

UML has been widely used for network system administrators, network system developers and users. UML is different from other virtualization technologies because it is a Virtual Operating System (OS), but to call UML can be done virtually. UML technologies such as VMWare are truly virtual machines by copying physical platforms, from the CPU to the device, even though the running OS on the physical platform also runs on the emulator platform provided by VMWare. In VMWare any OS that runs on a platform can be booted under VMWare, otherwise UML can only be a guest OS on Linux [4].

\subsection{Netkit}

Netkit is a place to set up and conduct network experiments at low cost and with little effort. The Netkit opensource network simulator was created by a group of university professors who use it as a tool in their teaching. It allows to create multiple virtual network devices (routers, switches, computers, etc.), which can be easily con- 
nected to form a network on one PC. The network equipment is virtual but has many original characteristics including the configuration interface [4-8].

\subsection{Linux iptables}

The Linux kernel's network packet processing subsystem is called Netfilter, and iptables is the command used to configure it. Iptables is software firewall that is included with most Linux distributions by default [9-10].

The iptables architecture groups network packet processing rules into tables by function (packet filtering, network address translation, and other packet mangling), each of which have chains (sequences) of processing rules. The rules consist of matches (used to determine which packets the rule will apply to) and targets (that determine what will be done with the matching packets). Iptables operates at OSI Layer 3 (Network). For OSI Layer 2 (Link), there are other technologies such as ebtables (Ethernet Bridge Tables) $[9,11]$.

\section{$3 \quad$ System Design Virtual Laboratory}

At this stage, the needs of computer hardware specifications used to design and implement virtual laboratories using Netkit will be explained. Hardware and software requirements are as follows:

- OS Debian 7, RAM 2 GB, Hardisk 320 GB, Processor Intel Dual Core

- Emulator Netkit (Netkit-2.8.tar.bz2, Netkit filesystemi386-F.2.tar.bz2, Netkitkernel-i386-K2.8.tar.bz2)

\subsection{Network topology}

Virtual lab experiments implementing iptables firewall security is depicted on the network topology implemented on Netkit, as shown in Fig. 1. 


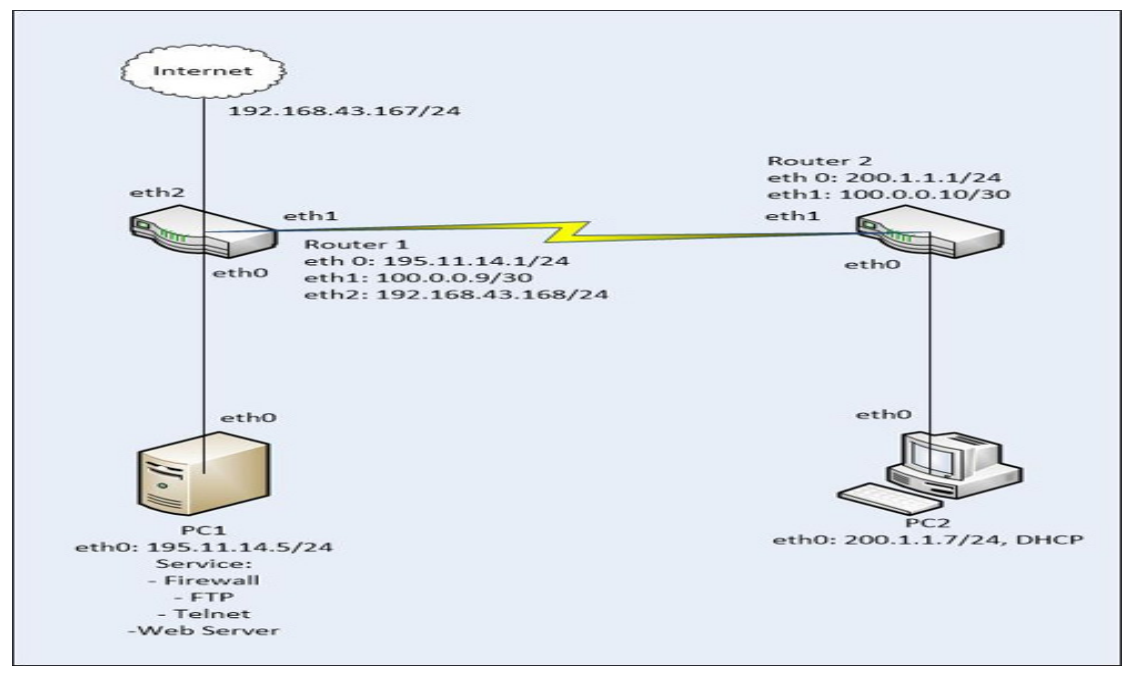

Fig. 1. Network Topology

The description of lab network topology at lab. linux server is shown in table 1.

Table 1. Design Details

\begin{tabular}{|c|l|l|c|}
\hline No & \multicolumn{1}{|c|}{ Computer Name } & \multicolumn{1}{c|}{ Lan Card } & Ip Address \\
\hline 1 & PC1 & eth0 & $195.11 .14 .5 / 24$ \\
\hline 2 & PC2 & eth0 & $200.1 .1 .7 / 24$ \\
\hline 3 & Router1 & eth0 & $195.11 .14 .1 / 24$ \\
\hline & Router1 & eth1 & $100.0 .0 .9 / 30$ \\
\hline & Router1 & eth2 & $192.168 .43 .168 / 24$ \\
\hline 4 & Router2 & eth0 & $100.1 .1 .1 / 24$ \\
\hline & Router2 & eth1 & $192.168 .43 .167 / 24$ \\
\hline 5 & Internet & eth0 & \\
\hline
\end{tabular}

\section{$4 \quad$ Virtual Laboratory Scenario}

At this stage, the lab is described. The virtual lab. linux server is created using netkit emulator. Virtual labs are implemented on a network basis with the network topology design in Fig. 1. The testing scenario of virtual laboratory implementation is shown in the following stages.

\subsection{Create a file lab.conf}

Create a lab.conf file is done to set the network scenario according to the network topology design the virtual lab. linux server, in accordance with the configuration syntax of the netkit emulator. The implementation of the lab.conf file is shown in Fig. 2. 


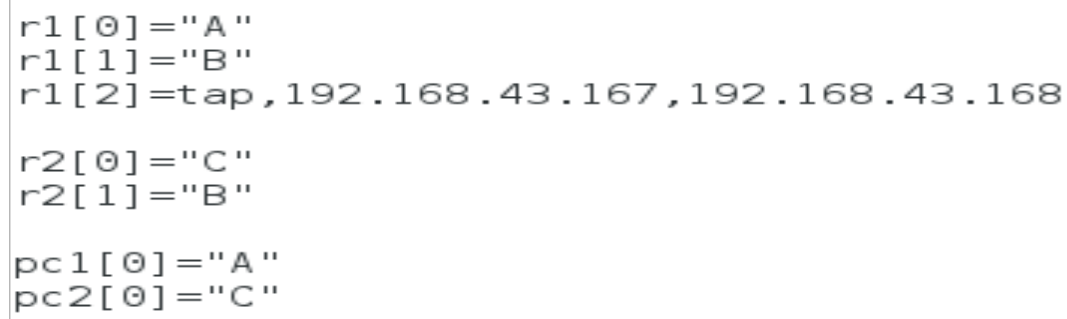

Fig. 2. lab.conf

In lab.conf to connect connections between NIC every computer is virtually used collosion domain. Collosion domains are symbols that connect virtually between NICs on a Netkit emulator. Implementation of the virtual lab. linux server consists of a virtual machine as a linux computer with scenario pc1, pc2, r1 and r2.

\subsection{Configure virtual machine (VM) in lab.conf}

At this stage, the writers configure the .startup file on Netkit. This file is required for the configuration and service to run when the virtual machine is started.

- Configuring VM pc1

Create a pc1.startup file is shown in Fig. 3.

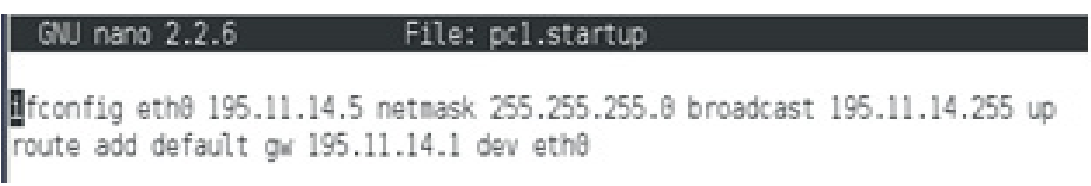

Fig. 3. A screenshot of pcl.startup

File and configuration for virtual machine are shown in Fig. 3, with ip address eth0 195.11.14.5/24 and route add ip gateway 195.11.14.1 with device eth0 therefore the pc1 virtual machine can be connected to the router $r 1$ virtual machine.

- Configuring VM pc2

Create a pc2.startup file is shown in Fig. 4.

GIJ nano 2.2.6 File: pc2.startup

Ifconfig ethe 260.1.1.7 netnask 255.255 .255 .8 broadcast 286.1.1.255 up route add default gw 200.1.1.1 dev ethe

Fig. 4. A screenshot of pc2.startup 
File and configuration for virtual machine are shown in Fig. 4, with ip address eth0 200.1.1.7/24 and route add ip gateway 200.1.1.1 with device eth0 therefore the pc2 virtual machine can be connected to the router $\mathrm{r} 2$ virtual machine.

- Configuring VM r1.startup

Create a r1.startup file is shown in Fig. 5.

GW nano 2.2.6 File: rl.startup

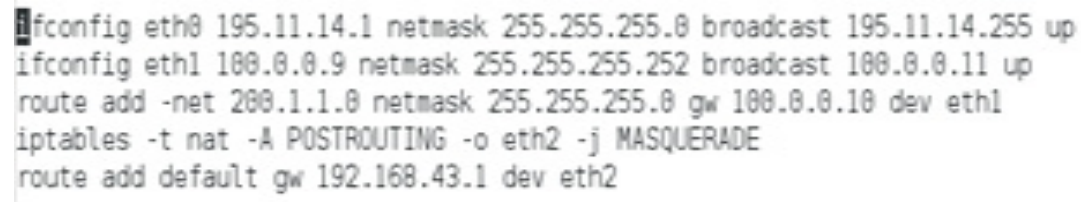

Fig. 5. A screenshot of r1.startup

File and configurations for router virtual machine $\mathrm{rl}$ are shown in Fig. 5, with ip address eth0 195.11.14.1/24, eth1 100.0.0.9/30 and route add network ip address 200.1.1.0/24 default gateway 100.0.10 with device eth1, so that $r$ can connect to $r 2$. Adds the command iptables -t nat -A Postrouting -o eth2 -j MASQUERADE, so eth2 devices can run and connect to the internet. Then the route command adds the standard gateway 192.168.43.1 with eth2 devices so that $\mathrm{r} 1$ can connect to the internet that passes through eth2 device.

- Configuring VM r2.startup

Create a r1.startup file is shown in Fig. 6 .

GIJ nano 2.2.6 File: r.startup

ifconfig ethe 200.1.1.1 netnask. 255.255 .255 .8 broadcast 200.1.1.255 up ifconfig eth1 109.6.6.18 netnask 255.255 .255 .252 broadcast 108.6.6.11 up route add -net 195.11 .14 .8 netnask $255.255 .255 .8 \mathrm{gw} 100.0 .0 .9$ dev ethl

Fig. 6. A screenshot of r2.startup

File and configurations for router virtual machine $\mathrm{r} 2$ are shown in Fig. 6, with ip address eth0 200.1.1.1/24, eth1 100.0.0.10/30 and route add network ip address 195.11.14.0/24 default gateway 100.0.0.9 with device eth1, so that $\mathrm{r} 2$ can connected to $\mathrm{r} 1$.

\section{$5 \quad$ Virtual Laboratory Implementation}

The implementation of the network topology design laboratory linux server Fig. 1. on the Netkit emulator is shown in Fig. 7. 


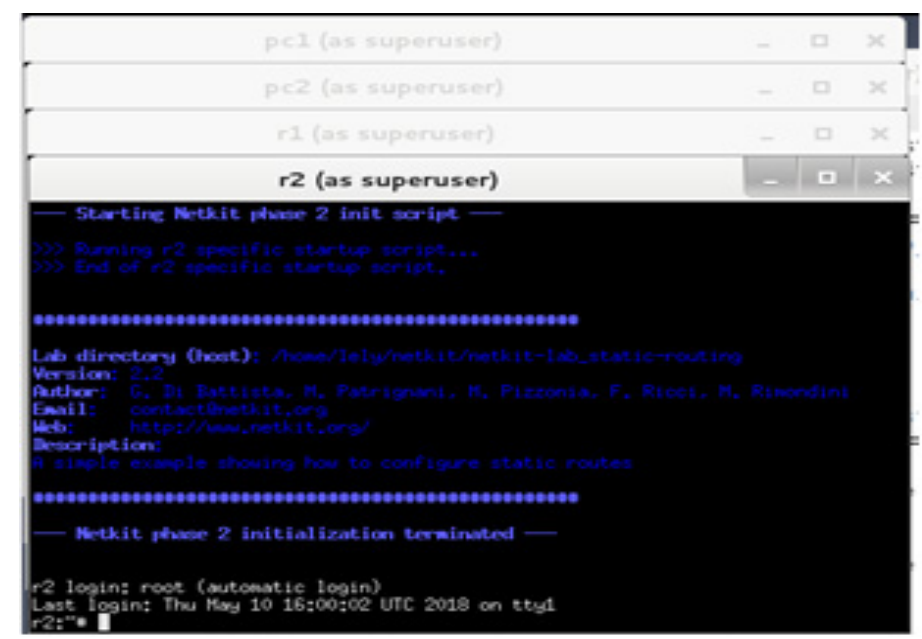

Fig. 7. Virtual Lab.Linux Server in Netkit Emulator

The results of virtual lab implementation are described as follows. Lab. Linux server is shown in Fig. 7. In the lab virtual machine ip address is configured.

Configuration checks that run on the virtual pcl.startup laboratory are shown in Fig. 8.

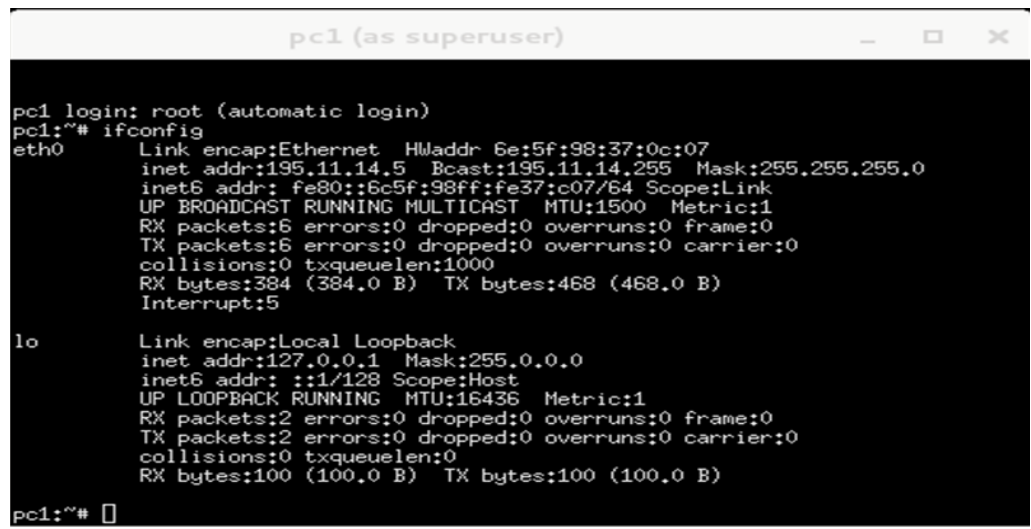

Fig. 8. Running Configuration pc1.startup

Configuration checks that run on virtual pc2.startup laboratories are shown in Fig.9. 


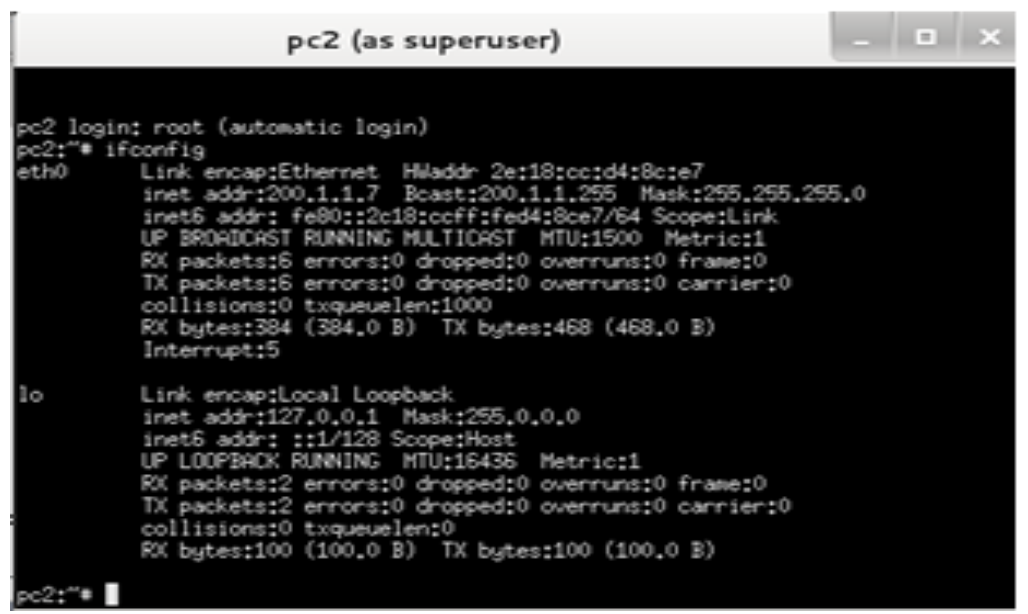

Fig. 9. Running Configuration pc2.startup

Configuration checks that run on virtual labs r1.startup are shown in Fig.10

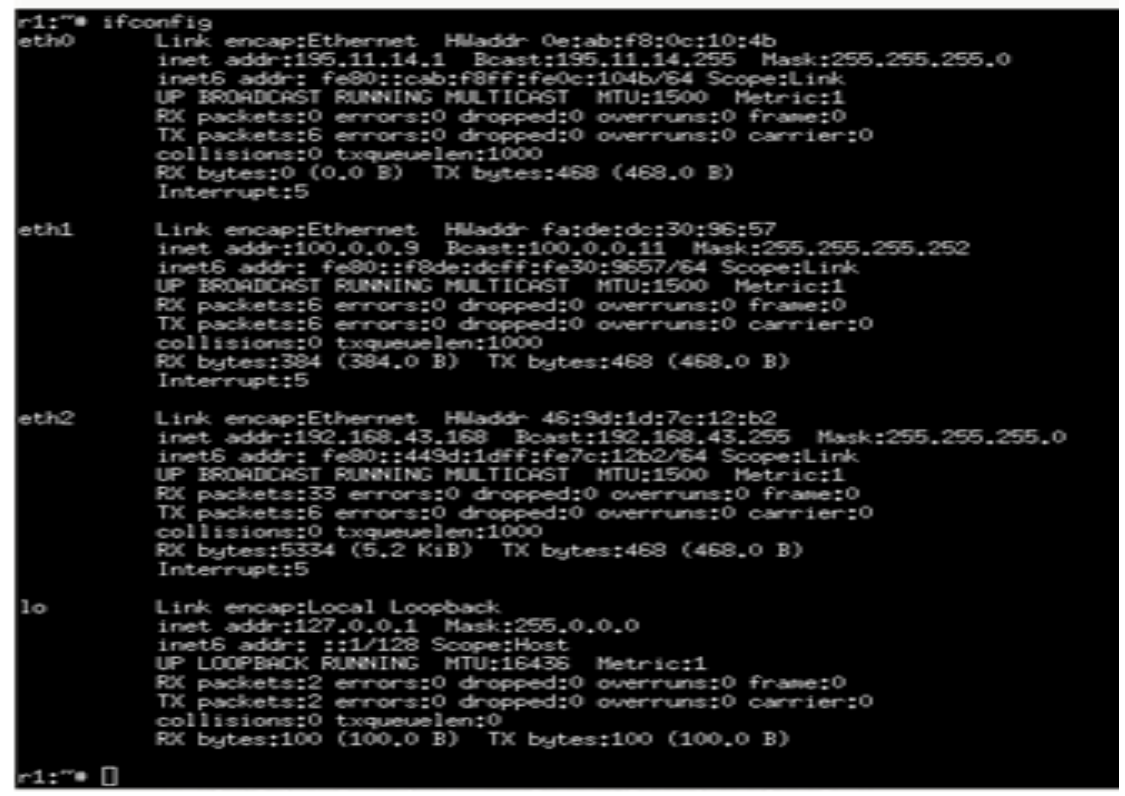

Fig. 10.Running Configuration r1.startup

Configuration checks that run on virtual lab r2.startup are shown in Fig. 11. 


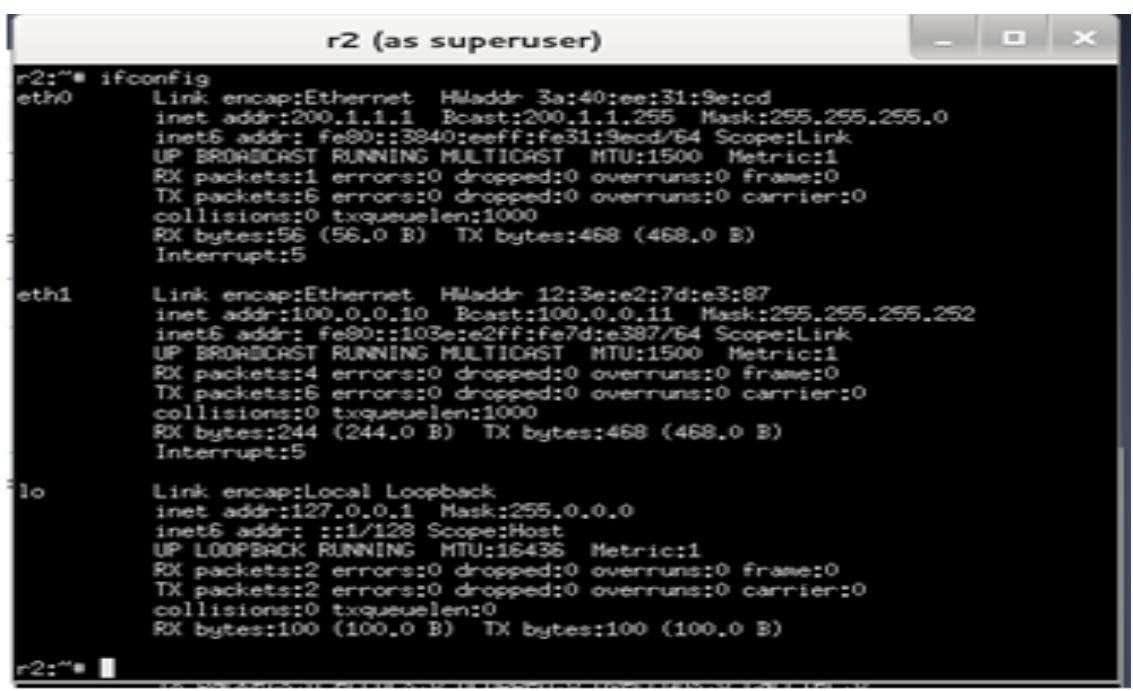

Fig. 11.Running Configuration r2.startup

\section{$6 \quad$ Testing and Analysis Virtual Laboratory Linux Server}

\subsection{Testing virtual laboratory linux server}

At this stage the virtual lab is tested where Linux server is run for FTP server, Telnet, web server, dhcp.

FTP server: To run the $\mathrm{ftp}$ server on $\mathrm{pc} 1$ and $\mathrm{pc} 2$, the command is executed to activate the ftp service, with the command \#/etc/init.d/proftpd restart.

FTP service testing is done by testing from PC2 to PC1 to start exchanging files. The test results are shown in Fig. 12, shown that the FTP connection is successful.

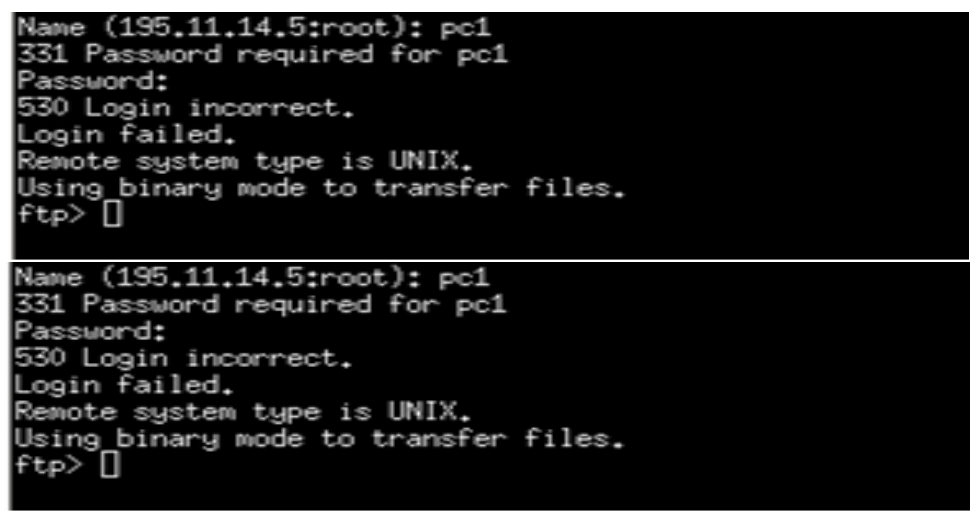

Fig. 12.FTP Server Running 
Telnet server: To run the telnet remote server on $\mathrm{pc} 1$ and $\mathrm{pc} 2$, the command is executed to activate the telnet service, with the command \#/etc/init.d/openbsd-inetd restart.

Remote telnet service testing is done by testing from PC2 to PC1 to start remote computer. Test results and telnet remote commands are shown in Fig. 13.

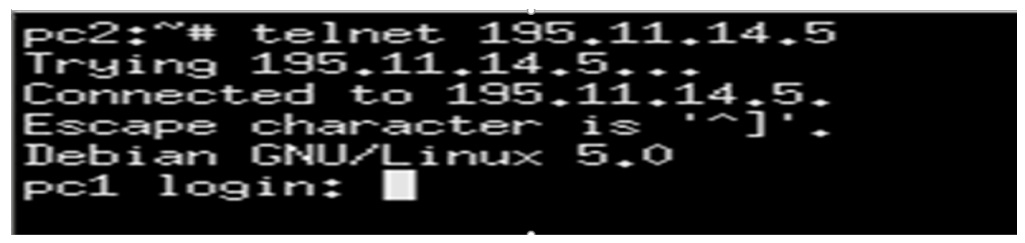

Fig. 13.Pc2 successfully remote Pc1

Web server: To run a web server on $\mathrm{PC} 1$, the command is executed to activate the web service server, with the command \# /etc /init.d /apache2 restart.

Web server testing is done by testing from $\mathrm{pc} 2$ to $\mathrm{pc} 1$ with the wget command to download the web page. The test results are shown in Fig. 14.

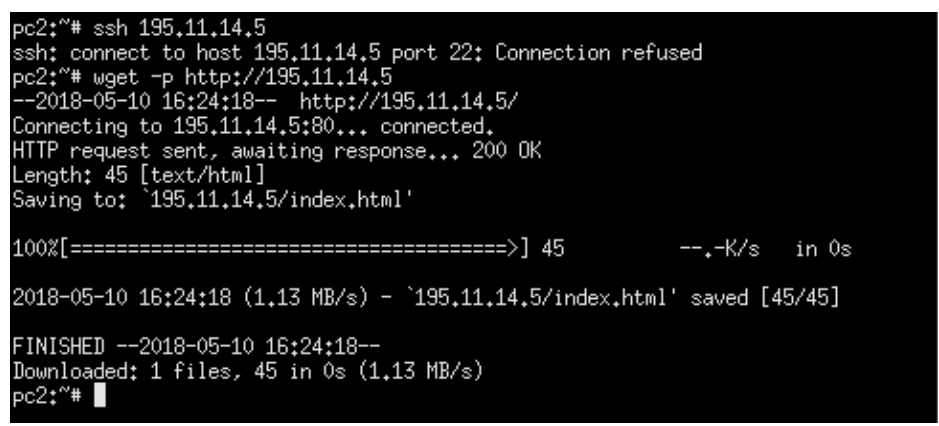

Fig. 14.Web access command use wget

DHCP server: To run dhcp server, configure $r 2$ as shown in Fig. 15. In $r 2$, it configures the IP Pool to be assigned to the DHCP Server. Then, the nano command is used in \#/etc/dhcp3/dhcpd.conf to change the dhcpd.conf file used to set the IP Pool on the DHCP Server. Range is an IP Address that can be used by the client. 


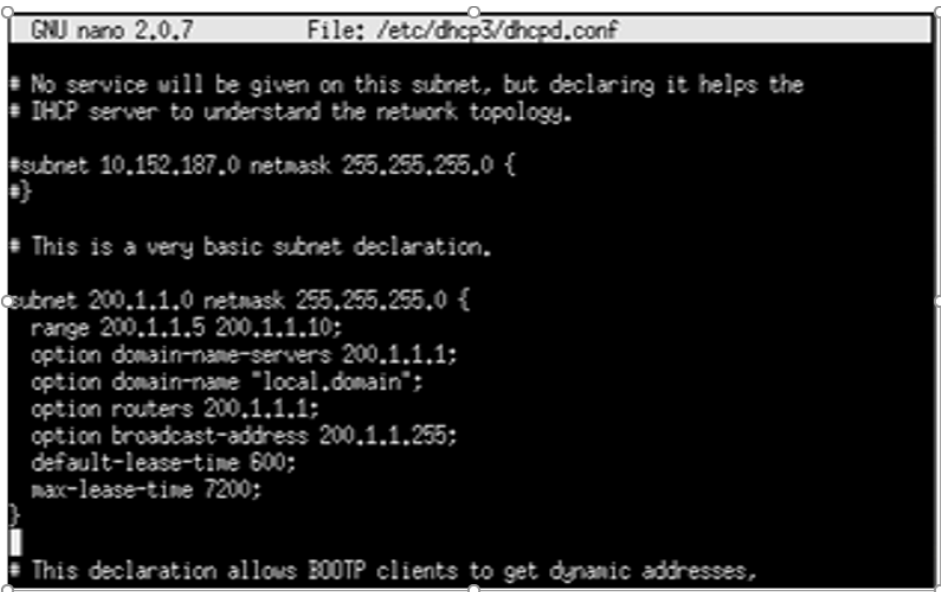

Fig. 15.Configure dhcp.conf

The next step, $\mathrm{r} 2$ does the domain setting in the resolv.conf file. To change the file, the nano command in /etc/resolv.conf which will be used to manage domains and nameservers on the DHCP Server is used. The configuration is shown in Fig. 16.

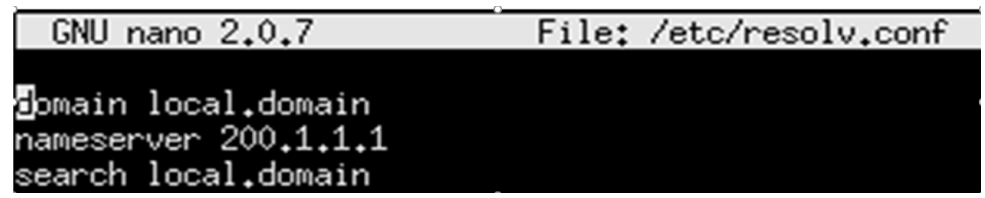

Fig. 16. Configure resolv.conf

The next step, $\mathrm{r} 2$ does the interface setting and use the nano command in / etc / default / dhcp3-server to change the interface to be used, DHCP Server, with interface = 'eth0'. The last step is restarting the DHCP server twice to make sure there are no errors in the DHCP settings. The command restarts the dhcp server service, with the command in \#/etc/init.d/dhcp3-server restart. To test dhcp server on pc2 which is connected with $\mathrm{r} 2$ is done by changing the configuration of pcm to dhcp. The configuration is shown in Fig. 17.

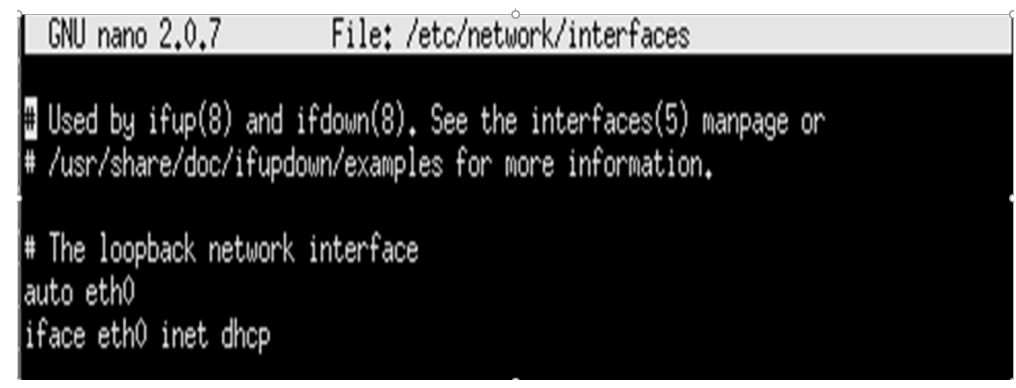


Fig. 17.Configuration Ip DHCP on Pc2.

The next step is to restart the network service on pc2 with command \#etc / init.d / networking restart. The last step is checking ip on pc2 from dhcp server $r 2$, as shown in Fig. 18.

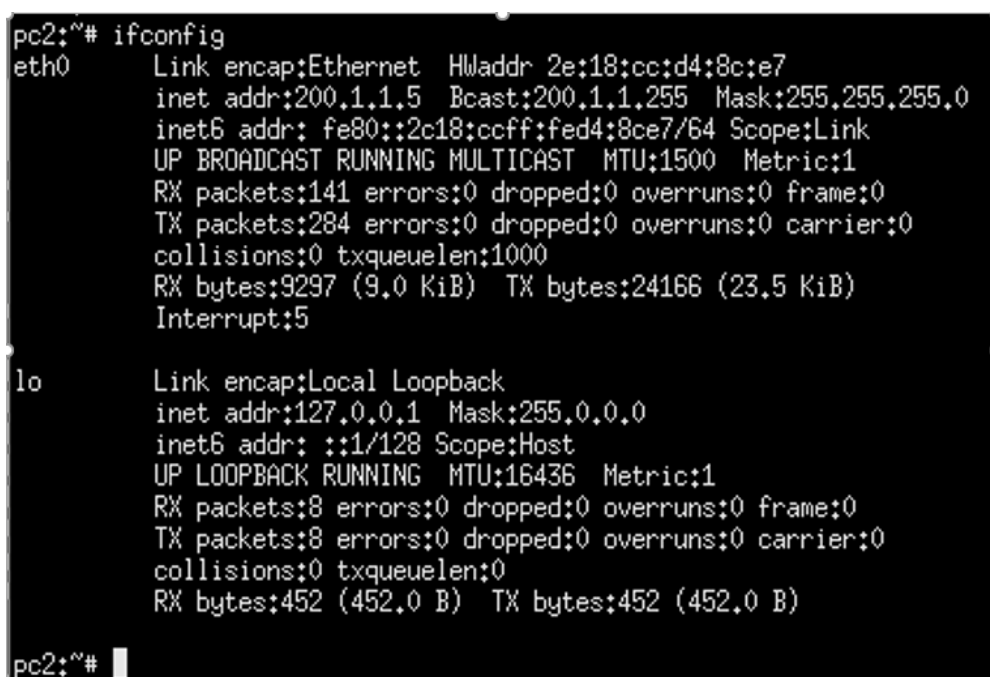

Fig. 18.Ipconfig Pc2

\subsection{Analysis virtual lab. linux server}

Analysis of virtual machine experiment in virtual Lab. Linux Server in table 2.

Table 2. Analysis of Linux Server Service Testing

\begin{tabular}{|l|l|l|l|l|}
\hline No & \multicolumn{1}{|c|}{ VM1 Client } & \multicolumn{1}{|c|}{ VM2 Server } & \multicolumn{1}{|c|}{ Service Server } & \multicolumn{1}{c|}{ Status } \\
\hline 1 & $\mathrm{pc} 2$ & $\mathrm{pc} 1$ & $\mathrm{ftp}$ & Running \\
\hline 2 & $\mathrm{pc} 2$ & $\mathrm{pc} 1$ & telnet & Running \\
\hline 3 & $\mathrm{pc} 2$ & $\mathrm{pc} 1$ & web & Running \\
\hline 4 & $\mathrm{pc} 2$ & $\mathrm{r} 2$ & $\mathrm{dhcp}$ & Running \\
\hline
\end{tabular}

\section{Conclusion}

From the test results, it can be concluded that all FTP, Telnet, Web and DHCP server on Linux can be run in a virtual lab. linux server on Netkit. The trials in the virtual laboratory shows the similarity of the work environment with real network systems, where Netkit can be implemented in a real network topology design. 


\section{$8 \quad$ References}

[1] Maurizio Pizzonial and Massimo Rimondini.Netkit: network emulation for education. SOFTWARE-PRACTICE AND EXPERIENCE Softw. Pract. Exper. 0000; 00:1-34 Published online in Wiley InterScience (www.interscience.wiley.com). https://doi.org/10.1002/spe.2273

[2] Paulo H. M. Gurgela*, Luiz H. C. Brancob, Ellen F. Barbosaa,Kalinka R. L. J. C. Brancoa. Development of a practical computer network course through Netkit virtualization tool. 2013 International Conference on Computational Science. Procedia Computer Science 18 ( 2013 ) 2583 - 2586. Publisher: Available online at www.sciencedirect.com. https://doi.org/10.1016/j.procs.2013.05.445

[3] Dike, Jeff. User Mode Linux. Prentice Hall, Apr 2006.

[4] University of Roma Tre Computer Networks Research Group. Netkit. http://www.netkit.org. accessed on 2 April 2018.

[5] Fermin Galan, David Fernandez, Javier Ruiz, Omar Walid, and Tomas de Miguel. Use of Virtualization Tools in Computer Network Laboratories. In Proc. 5th International Conference on Information Technology Based Higher Education and Training (ITHET 2004), pages 209\{214, Jun 2004. https://doi.org/10.1109/ithet.2004.1358165

[6] Maurizio Pizzonia, Massimo Rimondini. Easy Emulation of Complex Networks on Inexpensive Hardware. Proc. 4th International Conference on Testbeds and Research Infrastructures for the Development of Networks \& Communities (TRIDENTCOM 2008), Innsbruck, Mar 2008. https://doi.org/10.4108/tridentcom.2008.3155

[7] Massimo Rimondini. Emulation of Computer Networks with Netkit. Technical Report RTDIA-113-2007, Roma Tre University, Jan 2007.

[8] Hung Nguyen, Matthew Roughan, Simon Knight, Nick Falkner, Olaf Maennel, Randy Bush. How to Build Complex, Large-Scale Emulated Networks. Proc. 6th International Conference on Testbeds and Research Infrastructures for the Development of Networks \& Communities (TRIDENTCOM 2010), Berlin, May 2010. https://doi.org/10.1007/978-3642-17851-1_1

[9] Gregor N. Purdy. Linux Iptables Pocket Refences. O'Reilly.www.oreilly.com.2004.

[10] Qing-Xiu Wu. The Research and Application of Firewall based on Netfilter. 2012 International Conference on Solid State Devices and Materials Science. Procedia Computer Science 18 (2013) 2583 - 2586. Publisher: Available online at www.sciencedirect.com.

[11] Larry Peterson, Bruce Davie. Computer networks - A systems approach, 3rd Edition.Morgan Kauffman, 2004.

\section{$9 \quad$ Authors}

Yuri Ariyanto, State Polytechnic of Malang, Indonesia. Interst in research is network technology, cloud computing, security system and information technology.

Budi Harijanto, State Polytechnic of Malang, Indonesia. Interst in research is information systems.

Yan Watequlis S, State Polytechnic of Malang, Indonesia. Interst in research is information systems.

Article submitted 2018-10-31. Resubmitted 2019-03-13. Final acceptance 2019-03-14. Final version published as submitted by the authors. 